\title{
Nucleotide substitutions in the Candida albicans ERG11 gene of azole-susceptible and azole-resistant clinical isolates
}

\author{
Joanna Katarzyna Strzelczyk ${ }^{\bowtie}$, Anna Ślemp-Migiel ${ }^{2}$, Magdalena Rother ${ }^{1,3}$, Karolina Gołąbek ${ }^{1}$ \\ and Andrzej Wiczkowski ${ }^{1}$
}

'Chair and Department of General Biology, Medical University of Silesia, Zabrze, Poland; ${ }^{2}$ Laboratory of Microbiology in Nowy Targ Hospital, Nowy Targ, Poland; ${ }^{3}$ Postgraduate School of Molecular Medicine, Warsaw, Poland

\begin{abstract}
One of the mechanisms of Candida albicans resistance to azole drugs used in antifungal therapy relies on increased expression and presence of point mutations in the ERG11 gene that encodes sterol $14 a$ demethylase (14DM), an enzyme which is the primary target for the azole class of antifungals. The aim of the study was to analyze nucleotide substitutions in the Candida albicans ERG11 gene of azole-susceptible and azole-resistant clinical isolates. The Candida albicans isolates represented a collection of 122 strains selected from 658 strains isolated from different biological materials. Samples were obtained from hospitalized patients. Fluconazole susceptibility was tested in vitro using a microdilution assay. Candida albicans strains used in this study consisted of two groups: 61 of the isolates were susceptible to azoles and the 61 were resistant to azoles. Four overlapping regions of the ERG11 gene of the isolates of Candida albicans strains were amplified and sequenced. The MSSCP (multitemperature single strand conformation polymorphism) method was performed to select Candida albicans samples presenting genetic differences in the ERG11 gene fragments for subsequent sequence analysis. Based on the sequencing results we managed to detect 19 substitutions of nucleotides in the ERG11 gene fragments. Sequencing revealed 4 different alterations: T495A, A530C, G622A and A945C leading to changes in the corresponding amino acid sequence: D116E, K128T, V159I and E266D. The single nucleotide changes in the ERG11 gene did not affect the sensitivity of Candida albicans strains, whereas multiple nucleotide substitutions in the ERG11 gene fragments indicated a possible relation with the increase in resistance to azole drugs.
\end{abstract}

Key words: Candida albicans, ERG11 gene, sterol 14a demethylase, azole resistance

Received: 05 October, 2012; 25 June, 2013; 25 November, 2013 available on-line: 16 December, 2013

\section{INTRODUCTION}

Candida albicans is one of the most common opportunistic fungal human pathogens (Favre et al., 1999). In many healthy people it is a harmless colonizer of mucosal surfaces but very often it may induce diseases (Morschhäuser, 2002). This pathogenic yeast causes oral, vaginal and systemic infections mostly affecting immunocompromised patients, especially people who suffer from AIDS and bone marrow transplant recipients. In HIV (human immunodeficiency virus) positive patients oropharyngeal candidiasis (OPC) is frequently found (White et al., 2002). Candida albicans remains the prevalent reason of fungal infections diagnosed in hospitalized patients (Goldman et al., 2004).

Many categories of antifungal agents available for candidiasis treatment have been distinguished. The class of drugs that is most commonly used in therapy are azoles. Recently, we have observed an increasing failure in Candida infections treatment because of the emergence of resistance to antifungal drugs (Goldman et al., 2004). The condition of the patient's immune system, the drug characteristic and altered sensitivity of the fungus to the drugs are among the causes why antifungal therapy does not work properly (White et al., 2002). However, the resistance problems have arisen mainly from the overuse of antifungal agents and repeated antifungal therapies in chronic infections. Long-term or prophylactic treatment has given rise to Candida albicans resistant strains (Morschhäuser, 2002; Goldman et al., 2004).

Azoles are widely used in candidiasis due to their bioavailability and safety. They target the biosynthesis of ergosterol, the major sterol of the fungal membranes (Morschhäuser, 2002). Ergosterol is necessary to provide suitable integrity and functionality of the fungal membrane. Azoles inhibit competitively sterol $14 \alpha$ demethylase, a cytochrome P450 superfamily member, which is a key enzyme in the ergosterol biosynthesis pathway. The sterol $14 \alpha$ demethylase is responsible for the oxidative removal of the $14 \alpha$ methyl group from lanosterol. Azoles block the sterol $14 \alpha$ demethylase active centre by binding to it and making ergosterol synthesis and cell membrane formation impossible. This results in ergosterol depletion and accumulation of toxic ergosterol precursors which inhibit cell growth (Morschhäuser, 2002; Sanglard et al., 2003; Xu et al., 2008).

Resistance to azoles in Candida albicans yeast depends on multiple processes (Perea et al., 2001; Kamai et al., 2004). Mechanisms considered to be involved in azole resistance include the target enzyme alterations and the active efflux of antifungals (Sanglard et al., 2003). The most common mechanism is based on changing the target enzyme of the ergosterol biosynthesis pathway sterol $14 \alpha$ demethylase, which is encoded by the ERG11 gene. Resistance to azoles can be the result of both the increased expression of the ERG11 gene and the presence of point mutations. An increased expression of the

e-mail: asia.strzelczyk@gmail.com

Abbreviations: HIV, human immunodeficiency virus; 14DM, sterol 14 a demethylase; MSSCP, multitemperature single strand conformation polymorphism; OPC, oropharyngeal candidiasis; SSCP, single strand conformation polymorphism

*GenBank accession numbers: HM582441, HM582442, JX456529 
ERG11 gene leads to an increased intracellular production of the sterol $14 \alpha$ demethylase, which, in turn, increases the effective drug dose, whereas point mutations in the ERG11 gene cause amino acid substitutions and spatial configuration changes of ERG11p (sterol $14 \alpha$ demethylase) resulting in reduced affinity of the enzyme to azoles. Enhanced drug efflux is another widespread mechanism of azole resistance found in Candida albicans isolates. It is correlated with overexpression of genes which encode the two types of drug efflux transporters. The first are CDR1 and CDR2 genes encoding ABC (ATP-binding cassette) efflux pomp family and the second are MDR1 and FLU1 genes encoding members of the major facilitators superfamily (MFS) responsible for multidrug resistance. An increased expression of those genes in fungal cells account for the enhanced efflux of azoles and reduced intracellular accumulation of those drugs in fungal cells (Perea et al., 2001; Lupetti et al., 2002; Morschhäuser, 2002; Sanglard \& Odds, 2002; White et al., 2002; Maebashi et al., 2003; Goldman et al., 2004; Kamai et al., 2004; Akins, 2005; Xu et al., 2008).

The purpose of the present study was to evaluate the molecular mechanism of azole resistance related to alternations of the ERG11 gene in the collection of Candida albicans isolates resistant and susceptible to azoles. We attempted to detect some genetic differences as well as identify mutations present in the ERG11 gene that contribute to amino acid substitutions in sterol $14 \alpha$ demethylase, the target enzyme of the ergosterol synthesis pathway, in clinical samples of Candida albicans demonstrating the resistance and susceptibility to azoles.

\section{MATERIALS AND METHODS}

Materials. The Candida albicans isolates represented a collection of 122 strains C. albicans selected from 658 strains isolated from different biological materials (blood, urine, puss, sputum and bronchial swabs) from patients hospitalized in the John Paul II Memorial Independent Public Hospital in Nowy Targ. The Candida albicans strains were isolated from the clinical samples by culturing them on Sabouraud medium (Sabouraud Agara + $4 \%$ glucose) and Candida ID2 differential medium (bioMerieux, France). To confirm the correct identification of the Candida albicans isolates ID32C tests were applied (bioMerieux, France). Selected strains were stored as frozen stocks with $20 \%$ glycerol at $-80^{\circ} \mathrm{C}$. Before DNA extraction strains were subcultured on Sabouraud medium (Sabouraud Agara $+4 \%$ glucose) plates at $30^{\circ} \mathrm{C}$.

Selection of Candida albicans isolates susceptibile and resistant to azoles. MIC determinations. Susceptibility to fluconazole, itraconazole and voriconazole for strains isolated from the clinical materials was determined by the microdilution reference method using ATB Fungus 2 tests (bioMerieux, France) as recommended by the Clinical Laboratory and Standards Institute (CLSI) and the European Committee on Antimicrobial Susceptibility Testing (EUCAST). MICs were determined after $48 \mathrm{~h}$ of growth at $37^{\circ} \mathrm{C}$. MIC values for fluconazole, itraconazole and voriconazole were compared with the CLSI interpretative guidelines on antifungal susceptibility testing.

Strains showing $\mathrm{MIC} \leq 8 \mu \mathrm{g} \mathrm{ml} \mathrm{ml}^{-1}, \leq 0.125 \mu \mathrm{g} \mathrm{ml} \mathrm{ml}^{-1}$ and $\leq 1 \mu \mathrm{g} \mathrm{ml} \mathrm{m}^{-1}$ for fluconazole, itraconazole, and voriconazole, respectively, were considered as susceptible (S). Strains with MIC values for fluconazole $\geq 64 \mu \mathrm{g}$ $\mathrm{ml}^{-1}$, for itraconazole $\geq 2 \mu \mathrm{g} \mathrm{ml} \mathrm{m}^{-1}$, and for voriconazole $\geq 4 \mu \mathrm{g} \mathrm{ml} \mathrm{m}^{-1}$ were considered as resistant (R).

DNA extraction. Genomic DNA from the Candida albicans samples susceptible and resistant to azoles were extracted by Genomic Mini AX Yeast Kit (A\&A Biotechnology, Gdynia, Poland) according to the manufacturer's recommendation. DNA concentration was measured by spectrophotometric analysis with the use of GeneQuant (Amersham Biosciences, USA).

Polymerase Chain Reaction (PCR). Detection of Candida albicans ERG11 gene fragments was conducted by using the PCR technique. Amplifications were performed in $25 \mu \mathrm{l}$ reaction mixture under the following conditions: $12.5 \mu \mathrm{l}$ of PCR Master Mix, 2-fold concentration $\left(50 \mathrm{U} \mathrm{ml}^{-1}\right.$ of Taq polymerase supplied in a proprietary reaction buffer $\mathrm{pH} 8.5 ; 400 \mu \mathrm{M}$ each of dATP, dGTP, dCTP, dTTP; $3 \mathrm{mM} \mathrm{MgCl}$; Promega, USA); 2.5 $\mu \mathrm{l}$ of each primer at a concentration of $10 \mu \mathrm{M} ; 5 \mu \mathrm{l}$ of DNA and $2.5 \mu$ l of sterile water (Promega, USA).

4 primer sets were applied to amplify the Candida albicans ERG11 gene regions where missense point mutations are frequently observed. These point mutations were chosen on the basis of references (Favre et al., 1999). Primer sequences were designed by FastPCR Professional 5.2 Program (www.biocenter.helsinki.fi/bi/ Programs/download.htm). The primers were synthesized at the DNA Sequencing and Oligonucleotide Synthesis Laboratory at the Institute of Biochemistry and Biophysics, Polish Academy of Sciences, oligo.pl (Warsaw, Poland). The point mutations chosen for this study are presented in Table 1 and the primer sequences are described in Table 2.

The polymerase chain reaction was carried out in a Mastercycler Personal thermal cycler (Eppendorf, Germany). The thermal cycling conditions were as follows: 1 cycle at $94^{\circ} \mathrm{C}$ for $5 \mathrm{~min}, 35 \mathrm{cycles}$ at $95^{\circ} \mathrm{C}$ for $30 \mathrm{~s}, 60^{\circ} \mathrm{C}$ for $30 \mathrm{~s}$, and $72^{\circ} \mathrm{C}$ for $30 \mathrm{~s}$, and a final extension at $72^{\circ} \mathrm{C}$ for $4 \mathrm{~min}$.

Electrophoresis. The PCR products were resolved on $2 \%$ agarose gel (Sigma, USA) containing ethidium bromide (Serva, Germany). The electrophoresis was carried out for 60 minutes under the voltage of $100 \mathrm{~V}$ (Sigma Maxi Horizontal Gel Electrophoresis SHU 20 model, Sigma-Aldrich, Germany). The agarose gels were visualized under UV light and photographed (BIO-VISION, Vilber Lourmat, France). The GeneRuler 100 bp DNA Ladder was used as a molecular-weight size marker (Fermentas, Lithuania).

Table 1. Point mutations in ERG11 gene fragments (1C, 2C, 3C and 4C) chosen on the basis of reference (Favre et al., 1999).

\begin{tabular}{|c|c|c|c|c|c|c|c|}
\hline \multicolumn{2}{|c|}{$E R G 111 \mathrm{C}$} & \multicolumn{2}{|c|}{$E R G 11 \mathbf{2 C}$} & \multicolumn{2}{|c|}{$E R G 11 \mathbf{3 C}$} & \multicolumn{2}{|c|}{$E R G 114 \mathrm{C}$} \\
\hline${ }^{214}$ TTC-СТC & ${ }^{72}$ Phe-Leu & ${ }^{1348}$ GGG-GAG & ${ }^{450} \mathrm{Gly}$-Glu & 1213TCT-TाT & ${ }^{405}$ Ser-Phe & ${ }^{796} \mathrm{GAA}-\mathrm{GAC}$ & ${ }^{266} \mathrm{Glu}$-Asp \\
\hline${ }^{394}$ TAT-CAT & ${ }^{132} \mathrm{Tyr}-\mathrm{His}$ & ${ }^{1309} \mathrm{GTT}$-ATT & ${ }^{437}$ Val-Ile & & & & \\
\hline${ }^{375}$ TTC-TTA & 126Phe-Leu & ${ }^{1345}$ ТТТ-СТТ & 449Phe-Leu & & & & \\
\hline${ }^{346} \mathrm{GAT}-\mathrm{GAA}$ & ${ }^{116} \mathrm{Asp}-\mathrm{Glu}$ & & & & & & \\
\hline 394TAT-CAT & ${ }^{132} \mathrm{Tyr}-\mathrm{His}$ & & & & & & \\
\hline${ }^{427} \mathrm{AAA}-\mathrm{GAA}$ & ${ }^{143}$ Lys-Glu & & & & & & \\
\hline
\end{tabular}


Table 2. Primers used for ERG11 gene fragments amplification.

\begin{tabular}{|c|c|c|c|}
\hline $\begin{array}{l}\text { amplified } \\
\text { gene region }\end{array}$ & primer name & primer sequence & $\begin{array}{l}\text { PCR product } \\
\text { size }\end{array}$ \\
\hline \multirow{2}{*}{$1 C$} & ERG11-1CF & 5'-GCAGCTTCATATGGTCAACAACC-3' & \multirow{2}{*}{$325 \mathrm{bp}$} \\
\hline & ERG11-1CR & 5'-TAACATTGGCAACCCCATGAG-3' & \\
\hline \multirow{2}{*}{$2 \mathrm{C}$} & ERG11-2CF & 5'-GATACTGCTGCTGCCAAAGCTA-3' & \multirow{2}{*}{$265 b p$} \\
\hline & ERG11-2CR & 5'-CTGGTTCAGTAGGTAAAACCACCA -3' & \\
\hline \multirow{2}{*}{$3 C$} & ERG11-3CF & 5'-CTTCTGCTTCTACTTCTGCTTGG-3' & \multirow{2}{*}{$306 \mathrm{bp}$} \\
\hline & ERG11-3CR & 5'-CACTAGTATGAGCATAACCTG-3' & \\
\hline \multirow{2}{*}{$4 C$} & ERG11-4CF & 5'-TTGGAGACGTGATGCTGCTCAA-3' & \multirow{2}{*}{$211 b p$} \\
\hline & ERG11-4CR & 5'-AGCAGAAGTATGTGACCACCCA-3' & \\
\hline
\end{tabular}

Multitemperature single strand conformation polymorphism (MSSCP). The MSSCP technique was performed in order to select samples presenting genetic differences for subsequent sequence analysis. The MSSCP analysis was conducted by using a DNA Pointer apparatus. The DNA Pointer and all the reagents necessary to sample and gel preparation, and gel staining were provided by Kucharczyk T.E. (Warsaw, Poland). Electrophoresis was carried out on $8 \%$ polyacrylamide gels with $0.5 \mathrm{x}$ TBE (Tris-Borate-EDTA) buffer under the following conditions: $35-15-5^{\circ} \mathrm{C}$ temperature, $300 \mathrm{Vh}$ and $40 \mathrm{~W}$. Visualisation of DNA in polyacrylamide gels was performed by DNA/RNA Silver Stain Kit (Kucharczyk T.E., Warsaw, Poland) according to the manufacturer's recommendation. The GeneRuler 100 bp DNA Ladder (Fermentas, Lithuania) was used as a molecular-weight size marker (Slemp-Migiel et al., 2009).

ERG 11 gene sequencing. The nucleotide sequence analysis of both strands of the PCR products (1C, 2C, 3C and 4C) of the ERG11 gene was carried out at the DNA Sequencing Laboratory at the Institute of Biochemistry and Biophysics, Polish Academy of Sciences, oligo.pl (Warsaw, Poland) and at the Genomed Company (Warsaw, Poland). The PCR fragments were sequenced with an automated DNA Sequencer ABI3730 Genetic Analyzer (Applied Biosystems, USA) by ABI Prism $^{\text {TM }}$ BigDye ${ }^{\text {TM }}$ Terminator Cycle Sequencing kit by the standard protocols and previously designed primers. The nucleotide sequences of the ERG11 gene fragments from the tested strains were compared to the reference sequences of the ERG11 gene of Candida albicans strains downloaded from a database [GenBank accession numbers: X_13296 (NW_139492) at DNA level and XM_711729, XM_711668 at mRNA level].

Statistical analysis. Tests of Spearman rank correlation and $\chi^{2}$ test were used for statistical analysis. Significance level $p<0.05$ was adopted for the assessment of statistical significance.

\section{RESULTS}

\section{MIC determinations}

Depending on the MIC values obtained for azoles, the tested strains were divided into two extreme groups. The first group (group S) consisted of 61 Candida albicans strains susceptible to azole drugs (MIC for fluconazole was $<8 \mu \mathrm{g} \mathrm{ml} \mathrm{m}^{-1}$, for itraconazole $<0.125 \mu \mathrm{g} \mathrm{ml} \mathrm{m}^{-1}$ and for voriconazole $<1 \mu \mathrm{g} \mathrm{ml} \mathrm{m}^{-1}$ ). The second group consisted of 61 Candida albicans strains resistant to azoles (group R) and these isolates were characterized by a high resistance to fluconazole (MIC value $>128 \mu \mathrm{g} \mathrm{ml}^{-1}$ ), itraconazole (MIC value $>2 \mu \mathrm{g} \mathrm{ml}^{-1}$ ) and voriconazole (MIC value $>4 \mu \mathrm{g} \mathrm{ml}-1)$.

\section{PCR and MSSCP analysis}

The 1C, 2C, 3C and 4C ERG11 gene fragments were identified from the electrophoresis pattern of PCR products (Table 2).

Figs 1(a) and 1(b) show the MSSCP polyacrylamide gels with the separated amplification products of ERG11 gene fragments of Candida albicans strains. The MSSCP analysis revealed the presence of genetic differences in the 1C, 2C, 3C and 4C ERG11 gene fragments in Candida albicans tested strains sensitive and resistant to azoles as we described previously and was useful for selecting samples to sequence (Slemp-Migiel et al., 2009).

\section{Sequence analysis of ERG11 gene}

A thorough examination of the ERG11 gene fragment sequences in some previously chosen Candida albicans samples susceptible and resistant to azoles confirmed the nucleotide substitutions in the examined gene fragments. Substitutions of nucleotides in the ERG11 gene fragments were detected in both groups. We managed to detect the following point mutations in the analyzed yeast isolates: C216T, A354G, T462C, T495A, A504G, A530C, C558T and G622A mutations in the 1C ERG11 gene fragment. In the 2C ERG11 gene fragment we observed the following mutations: G1309A, A1587G and T1617C. T1143C, A1167G, A1173G, T1203C, A1230G, T1257C and T1287C mutations were observed in the 3C ERG11 gene fragment. We also detected a A945C mutation in the 4C ERG11 gene fragment. These point mutations characteristic of Candida albicans strains sensitive and resistant to azoles are presented in Table 3.

Based upon the sequencing results of ERG11 gene fragments obtained from Candida albicans isolates the molecular changes in sterol $14 \alpha$ demethylase protein were deduced. Some of the point mutations detected in ERG11 gene fragments led to the following amino acid substitutions: D116E, K128T, V159I and E266D. These 

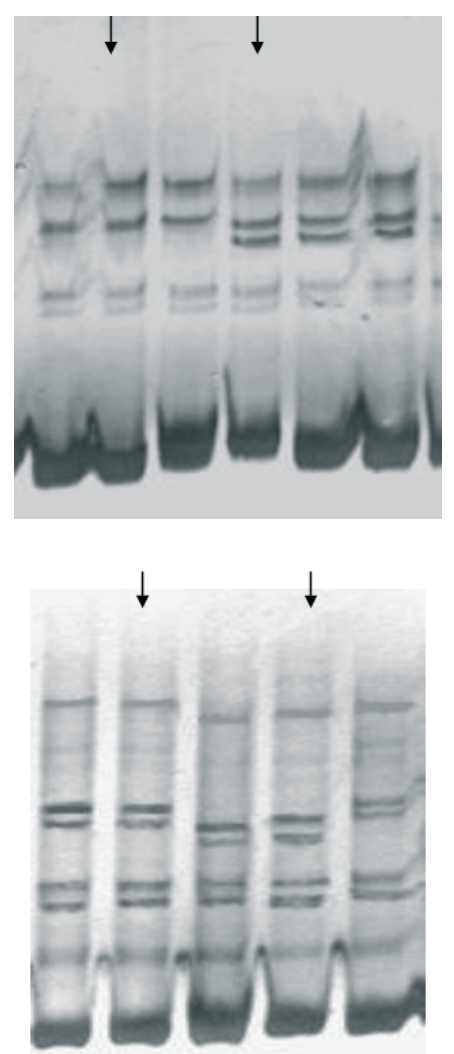

Figure $1 \mathrm{a}$ and $\mathrm{b}$. Polyacrylamide gels with Candida albicans sensitive and resistant to azoles MSSCP analysis of the ERG11 gene fragments amplification products.

The arrows indicate the MSSCP electrophoretic profiles which clearly differ from the others.

amino acid alterations were the consequence of T495A, A530C, G622A and A945C mutations, respectively. The point mutations in the ERG11 gene and amino acid substitutions in ERG11p (sterol $14 \alpha$ demethylase) corresponding to them are presented in Table 4.

\section{DISCUSSION}

The main mechanisms of fluconazole resistance in Candida albicans are changes in the target enzyme, sterol $14 \alpha$ demethylase, due to mutations in the encoding gene, ERG11, increased expression of ERG11, and upregulation of the efflux pumps that transport fluconazole and other toxic compouds out of the cell (MacCallum et al., 2010; Sasse et al., 2012). Furthermore, several of these mechanisms e.g., combination of mutations in MRR1, TAC1 and ERG11 genes (Sasse et al., 2012) may operate simultaneously to result in high levels of fluconazole resistance. Chen et al. (2010) suggested that increased expression of the CDR1 and CDR2 genes may play an important role in fluconazole-resistant strains of Candida albicans with G487T and T916C mutations in the ERG11 gene.

PCR amplification and sequencing of the ERG11 gene fragments showed 19 nucleotide changes in Candida albicans isolates. Some of the mutations presented in our results were similar to those reported previously (Franz et al., 1998; Marr et. al., 1998; Sanglard et al., 1998; Favre et al., 1999; Perea et al., 2001; Martinez et al., 2002; Maebashi et al., 2003; Kamai et al., 2004; Xu et al., 2008). The remaining mutations: A354G, G622A and T1287C revealed in the nucleotide sequence analysis of the ERG11 gene were, to the best of our knowledge, not referenced in the literature. They are novel alterations that we presented for the first time. The A354G and T1287C mutations are probably silent substitutions because it has not been proven that they may lead to changes in the protein level.

Two of the mutations detected by us in the ERG11 gene: C558T and A354G, were prevalent only in Candida albicans isolates sensitive to azoles (Table 3). In a study described by Marr et al. (2010) the C558'T substitution was present in both the sensitive and resistant isolates of Candida albicans, thus, the mutation is not related to fluconazole resistance. The second mutation present only in sensitive strains, A354G, has not been described previously. As mentioned above, we did not manage to retrieve its verification in the available references.

Five genetic alterations in ERG11 were identified only in resistant isolates: A530C, G622A, G1309A, A1167G, A1230G. Among these mutations the G1309A substitution appeared frequently (54\%) while A1230G was rarely observed $(6.5 \%)$ ('Table 3$)$. It should be noted that $33 \%$ (20/61) of resistant isolates examined in our study had the same three substitutions: A530C, G622A, A1167G. Two of them, A530C and G622A, led to amino acid changes in ERG11p, suggesting that the resistant phenotype in these isolates could be caused by alterations in the ERG11 gene. More than 140 different amino acid substitutions in ERG11p of Candida albicans clinical isolates have been reported. This high genetic polymorphism suggests that ERG11 is highly permissive to structural changes (Morio et al., 2010). We discovered the T495A, A530C, G622A and A945C mutations in the ERG11 gene, which turned out to be the most significant in raising the resistance because they led to amino acid substitutions in ERG11p (Table 4). Nucleotide substitution of T495A caused the exchange of glutamate for aspartate in position 116 (D116E) in sterol $14 \alpha$ demethylase. The A530C mutation contributed to protein changing by replacing lysine with arginine in position 128 (K128T). The G622A mutation caused a change from valine to isoleucine in the 159 position (V159I) and the A945C mutation replaced glutamate with aspartate in the 266 position (E266D). It has been reported that amino acid substitutions in ERG11p cause changes in the azole affinity of the enzyme (Kamai et al., 2004). Mutations in ERG11p are clustered in three regions: between amino acids 105 to 165 near the $\mathrm{N}$-terminus of the protein, and amino acids 266-287 and 405-488 situated towards the $\mathrm{C}$ terminus of the enzyme, which are suggested to be the hotspot regions (Marichal et al., 1999; Maebashi et al., 2003; Kamai et al., 2004; Wang et al., 2009). All the amino acid alterations detected by us are clustered in these three regions of ERG11p.

Sanglard et al. (1998) and Perea et al. (2001) revealed that the T348A mutation appeared both in azole susceptible and resistant strains and caused the D116E amino acid substitution. Favre et al. (1999) also proved the presence of the D116E change as a result of the T348A mutation in Candida albicans resistant strains. In our research the D116E amino acid alteration was an effect of the T495A mutation at the nucleotide level (according to X_13296 reference sequence). T495A is the same mutation as T348A (according to XM_711729 reference sequence). Our surveys demonstrated the same results as the results of sequencing reported by $\mathrm{Xu}$ et al. (2008). Our data showed a high frequency of the T495A (D116E) substitution. In fact, we observed this mutation in $61 \%$ of azole sensitive isolates and in 33\% of azole resistant isolates (Table 3). Perea et al. (2001) found the D116E substitution to be associated with a resistant phe- 
Table 3. Nucleotide substitutions in the ERG11 gene of Candida albicans strains sensitive and resistant to azoles in comparison with the published sequence [GenBank accession numbers: X_13296 (NW_139492) at DNA level and XM_711729, XM_711668 at mRNA level].

\begin{tabular}{|c|c|c|}
\hline \multirow[b]{2}{*}{ ERG11 gene mutations } & \multicolumn{2}{|c|}{ Number of strains } \\
\hline & $\begin{array}{l}\text { C. albicans sensitive } \mathrm{MIC} \text { values } \\
\text { fluconazole }<8 \mu \mathrm{g} \mathrm{ml}-1 \\
\text { itraconazole }<0.125 \mathrm{\mu g} \mathrm{ml}^{-1} \\
\text { voriconazole }<1 \mu \mathrm{g} \mathrm{ml}-1\end{array}$ & $\begin{array}{l}\text { C. albicans resistant MIC values } \\
\text { fluconazole }>128 \mu \mathrm{g} \mathrm{ml}^{-1} \\
\text { itraconazole }>2 \mu \mathrm{g} \mathrm{ml}^{-1} \\
\text { voriconazole }>4 \mu \mathrm{g} \mathrm{ml}^{-1}\end{array}$ \\
\hline C216T & $16(26 \%)$ & $4(6.5 \%)$ \\
\hline A354G & $37(61 \%)$ & $0(0 \%)$ \\
\hline $\mathrm{T} 462 \mathrm{C}$ & $53(87 \%)$ & $33(54 \%)$ \\
\hline T495A & $37(61 \%)$ & $20(33 \%)$ \\
\hline A504G & 37 (61\%) & $20(33 \%)$ \\
\hline A530C & $0(0 \%)$ & $20(33 \%)$ \\
\hline $\mathrm{C} 558 \mathrm{~T}$ & $53(87 \%)$ & $0(0 \%)$ \\
\hline G622A & $0(0 \%)$ & $20(33 \%)$ \\
\hline G1309A & $0(0 \%)$ & $33(54 \%)$ \\
\hline A1587G & $32(52 \%)$ & $37(61 \%)$ \\
\hline $\mathrm{T} 1617 \mathrm{C}$ & $32(52 \%)$ & $34(56 \%)$ \\
\hline T1143C & $32(52 \%)$ & $29(47.5 \%)$ \\
\hline A1167G & $0(0 \%)$ & $24(39 \%)$ \\
\hline A1173G & $32(52 \%)$ & $29(47.5 \%)$ \\
\hline T1203C & $32(52 \%)$ & $29(47.5 \%)$ \\
\hline A1230G & $0(0 \%)$ & $4(6.5 \%)$ \\
\hline $\mathrm{C} 1257 \mathrm{~T}$ & $52(85 \%)$ & $20(33 \%)$ \\
\hline T1287C & 36 (59\%) & 36 (59\%) \\
\hline A945C & 30 (49\%) & 7 (11\%) \\
\hline
\end{tabular}

notype but according to Sanglard et al. (1998) and Xu et al. (2008) this molecular change is not likely to represent mutation linked with an azole antifungal agent resistant phenotype.

The results of the ERG11 gene sequence analysis displayed the presence of the A530C mutation in our isolates leading to the K128T change in the corresponding amino acid sequence. The same outcome in Candida albicans isolates was presented by $\mathrm{Xu}$ et al. (2008). Sanglard et al. (1998) and Perea et al. (2001) also confirmed the K128T amino acid alteration in sterol $14 \alpha$ demethylase which was a consequence of the A383C mutation according to XM_711729 (this is the same mutation as A530C according to the X_13296 reference sequence). In our study this mutation characterized only Candida albicans isolates resistant to azoles it was present in 33\% of the resistant isolates (results presented in Table 3). Another study did not yield the same result as ours. The K128T amino acid substitution was detected by Perea $e t$ al. (2001) both in susceptible and resistant isolates and

Table 4. Mutations leading to amino acid substitutions in sterol $14 a$ demethylase.

\begin{tabular}{ll}
\hline ERG11 gene mutations & Amino acid substitutions \\
\hline T495A & D116E \\
\hline A530C & K128T \\
\hline G622A & V159I \\
\hline A945C & E266D \\
\hline
\end{tabular}

according to the authors this substitution was not associated with azole resistance. The K128T amino acid substitution was confirmed not to participate in azole resistance also by Sanglard et al. (1998), Favre et al. (1999), and Xu et al. (2008). On the other hand, the D116E and K128T amino acid alterations are located in $14 \alpha$ sterol demethylase in the region between the B' and C helices. Which was suggested to be involved in inhibitor or substrate-elicited structural lesions (Goldman et al., 2004). Thus, these substitutions may be relevant for the development of resistance.

In our study, the DNA sequencing of the ERG11 gene revealed a G622A substitution which entailed a V159I amino acid change. We found this mutation only in Candida albicans isolates resistant to azoles $(33 \%)$ (Table 3). This mutation lies exactly in the first hot spot of the sterol $14 \alpha$ demethylase protein (amino acid position 105-165 identified by Marichal et al., 1999) and may be associated with azole resistance. Morio et al. (2010) described amino acid substitution V159I in sterol $14 \alpha$ demethylase but only in azole-susceptible isolates of Candida albicans, This is probably the same substitution as the one identified by us but we could not find any information about corresponding changes at the nucleotide level. Moreover, substitution V159I was rarely described in literature, on the contrary, the D116E, K128T, E266D substitutions were reported frequently (Morio et al., 2010).

Some authors indicated the important role of the A798C mutation (the same as A945C according to X_13296) in the ERG11 gene which might result in the replacement of glutamate by aspartate (E266D) in sterol $14 \alpha$ demethylase and lower affinity of the enzyme to azoles (Favre et al., 1999; Lee et al., 2004). The E266D amino acid alteration is in the G-helix of the enzyme which partly covers the active site of sterol $14 \alpha$ demethylase (Maebashi et al., 2003). The E266D alteration was demonstrated by us as an outcome of the A945C nucleotide change and the mutation appeared mostly in sensitive strains $(49 \%)$ and only in $11 \%$ of the resistant strains (Table 3). The same alteration was observed by White et al. (2002) in azole susceptible and resistant isolates, while Franz et al. (1998) and Xu et al. (2008) observed the E266D amino acid change only in susceptible isolates. According to the authors the E266D mutation cannot cause fluconazole resistance. Also Favre et al. (1999) agree that the E266D substitution found in the azole-resistant isolates seems to be irrelevant for conferring resistance to azoles.

It is worth noting that in our research $33 \%$ (20/61) of resistant isolates had the same three substitutions: T495A, A530C, G622A, which resulted in amino acid substitutions D116E and K128T, V159I, respectively. Overall, Maebashi et al. (2003) reported two amino acid substitu- 
tions in ERG11, namely E266D and V488I, which contributed to the increased fluconazole resistance. On the contrary, a single amino acid change of E266D was not related to the decreased susceptibility to the drug. The occurrence of two or more amino acid substitutions appears to be common in ERG11 from fluconazole-resistant strains and some mutations can have an effect only when combined with others (Favre et al., 1999, Xu et. al., 2008, Morio et al., 2010). The ERG11 substitutions are linked to azole resistance but the presence of ERG11 mutations in sensitive strains is not easily translated. Resistance formation requires long-term exposure to an azole drug, however previous studies have not related mutations in sensitive isolates to fluconazole exposure (Wang et al., 2009).

Amino acid alterations D116E, K128T, V159I and E266D detected in our studies were distributed heterogeneously in all investigated resistant isolates of Candida albicans. A change from heterozygosity to homozygosity for the ERG11 gene was described to be responsible for the increased fluconazole resistance (Franz et al., 1998; Chau et al., 2004). The homozygosity of mutations within this gene was suggested to enhance azole resistance (Maebashi et al., 2003; Kamai et al., 2004). The E266D change does not contribute to resistance, since an enzyme with reduced affinity for fluconazole should confer a resistant phenotype in the heterozygous state (Franz et al., 1998). Our one susceptible strain was homozygous for the E266D amino acid alteration but the lack of fluconazole resistant growth may be due to the presence of only this one mutation in that isolate (Favre et al., 1999; Xu et al., 2008).

Candida albicans is a diploid organism. The mutations usually occur randomly on each allele and result in heterozygosity. Meiosis, frequent mitotic recombination, mating and gene conversion may bring about homozygosity of the altered gene. Mitotic recombination or gene conversion is a probable explanation for ERG11 homozygosity in Candida albicans resistant isolates. Accumulated homozygous nucleotide changes leading to amino acid substitutions within the hot spot regions of ERG11p contributed to the increased azole resistance (Lee et al., 2004). ERG11 polymorphisms may play a role in increasing the level of resistance but do not cause it directly (Wang et al., 2009). Point mutations in the ERG11 gene in the isolates could be associated with resistance or they could be the result of allelic variations between different strains of Candida albicans (White et al., 2002).

Besides the ERG11 pathway changes there might exist other mechanisms responsible for azole resistance such as increased expression of the ERG11 gene or of the CDR drug efflux pumps (Rex et al., 1995; White et al., 2002; Prasad \& Kapoor, 2005). It is considered that Candida albicans infection therapy with azoles resulted in resistant strains selection. Those strains demonstrated more than one point mutation in the ERG11 gene or even more than one resistance mechanism in cells (Owen et al., 2000; Perea et al., 2001; Chau et al., 2004). A better understanding of the resistance mechanism as well as its early detection are essential for patient management (White et al. 1998; Morschhäuser, 2002).

\section{REFERENCES}

Akins RA (2005) An update on antifungal targets and mechanisms of resistance in Candida albicans. Med Mycol 43: 285-318.

Chau AS et al. (2004) Application of real-time quantitative PCR to molecular analysis of Candida albicans strains exhibiting reduced susceptibility to azoles. Antimicrob Agents Chemother 48: 2124-2131.

Chen LM et al. (2010) Overexpression of CDR1 and CDR2 genes plays an important role in fluconazole resistance in Candida albicans with G487T and T916C mutations. J Int Med Res 38: 536-45.
Favre B, Didmon M, Ryder NS (1999) Multiple amino acid substitutions in lanosterol 14alpha-demethylase contribute to azole resistance in Candida albicans. Microbiology 145: 2715-2725.

Franz R et al. (1998) Multiple molecular mechanisms contribute to a stepwise development of fluconazole resistance in clinical Candida albicans strains. Antimicrob Agents Chemother 42: 3065-3072.

Goldman GH et al. (2004) Evaluation of fluconazole resistance mechanisms in Candida albicans clinical isolates from HIV-infected patients in Brazil. Diagn Microbiol Infect Dis 50: 25-32.

Kamai Y et al. (2004) Characterization of mechanisms of fluconazole resistance in a Candida albicans isolate from a Japanese patient with chronic mucocutaneous candidiasis. Microbiol Immunol 48: 937-943.

Lee MK et al. (2004) Drug resistance genes and trailing growth in Candida albicans isolates. I Antimicrob Chemother 53: 217-224.

Lupetti A et al. (2002) Molecular basis of resistance to azole antifungals. Trends Mol Med 8: 76-81.

MacCallum DM et al. (2010) Genetic dissection of azole resistance mechanisms in Candida albicans and their validation in a mouse model of disseminated infection. Antimicrob Agents Chemother 54: 147683.

Maebashi K et al. (2003) Proliferation of intracellular structure corresponding to reduced affinity of fluconazole for cytochrome P-450 in two low-susceptibility strains of Candida albicans isolated from a Japanese AIDS patient. Microbiol Immunol 47: 117-124.

Marichal P et al. (1999) Contribution of mutations in the cytochrome P450 14 $\alpha$-demethylase (Erg11p, Cyp51p) to azole resistance in Candida albicans. Microbiology 145: 2701-2713.

Marr KA et al. (1998) Rapid, transient fluconazole resistance in Candida albicans is associated with increased mRNA levels of CDR. Antimicrob Agents Chemother 42: 2584-2587.

Martinez M et al. (2002) Heterogeneous mechanisms of azole resistance in Candida albicans clinical isolates from an HIV-infected patient on continuous fluconazole therapy for oropharyngeal candidosis. $J A n$ timicrob Chemother 49: 515-524.

Morio F et al. (2010) Screening for amino acid substitutions in the Candida albicans Erg11 protein of azole-susceptible and azole-resistant clinical isolates: new substitutions and a review of the literature. $D i$ agn Microbiol Infect Dis 66: 373-84.

Morschhäuser J (2002) The genetic basis of fluconazole resistance development in Candida albicans. Biochim Biophys Acta 1587: 240-248.

Owen LE et al. (2000) Evolution of drug resistance in experimential populations of Candida albicans. I Bacteriol 182: 1515-1522.

Perea S et al. (2001) Prevalence of molecular mechanisms of resistance to azole antifungal agents in Candida albicans strains displaying highlevel fluconazole resistance isolated from human human immunodeficiency virus-infected patients. Antimicrob Agents Chemother 45: 2676-2684.

Prasad R, Kapoor K (2005) Multidrag resistance in yeast Candida. Infect Rev Cytol 242: 215-248.

Rex JH, Rinaldi MG, Pfaller MA (1995) Resistance of Candida species to fluconazole. Antimicrob Agents Chemother 39: 1-8.

Sanglard D et al. (1998) Amino acid substitutions in the cytochrome P-450 lanosterol 14 $\alpha$-demethylase (CYP51A1) from azole-resistant Candida albicans clinical isolates contribute to resistance to azole antifungal agents. Antimicrob Agents Chemother 42: 241-253.

Sanglard D, Odds FC (2002) Resistance of Candida species to antifungal agents: molecular mechanisms and clinical consequences. Lancet Infect Dis 2: 73-85.

Sanglard D et al. (2003) Candida albicans mutations in the ergosterol biosynthetic pathway and resistance to several antifungal agents. Antimicrob Agents Chemother 47: 2404-2412.

Sasse C, Dunkel N, Schäfer T, Schneider S, Dierolf F, Ohlsen K, Morschhäuser J (2012) The stepwise acquisition of fluconazole resistance mutations causes a gradual loss of fitness in Candida albicans. Mol Microbiol 86: 539-56.

Slemp-Migiel A et al. (2009) MSSCP technology as a tool for the detection of genetic differences in ERG11 gene in Candida albicans sensitive and resistant to azoles. Med Mycol 16: 5-9.

Wang H (2009) Rapid detection of ERG11 gene mutations in clinical Candida albicans isolates with reduced susceptibility to fluconazole by rolling circle amplification and DNA sequencing. BMC Microbiol 9: 167-178.

White TC, Marr KA, Bowden RA (1998) Clinical, cellular, and molecular factors that contribute to antifungal drug resistance. Clin Microbiol Rev 11: 382-402.

White TC et al. (2002) Resistance mechanisms in clinical isolates of Candida albicans. Antimicrob Agents Chemother 46: 1704-1713.

$\mathrm{Xu} \mathrm{Y,} \mathrm{Chen} \mathrm{L,} \mathrm{Li} \mathrm{C} \mathrm{(2008)} \mathrm{Susceptibility} \mathrm{of} \mathrm{clinical} \mathrm{isolates} \mathrm{of} \mathrm{Candida}$ species to fluconazole and detection of Candida albicans ERG11 mutations. J Antimicrob Chemother 61: 798-804. 